\title{
Sulfur in the globular clusters 47 Tucanae and NGC 6752 ${ }^{\star}$
}

\author{
L. Sbordone ${ }^{1,2}$, M. Limongi ${ }^{3}$, A. Chieffi ${ }^{4}$, E. Caffau ${ }^{2}$, H.-G. Ludwig ${ }^{1,2}$, and P. Bonifacio ${ }^{1,2,5}$ \\ 1 CIFIST Marie Curie Excellence Team, France \\ e-mail: luca. sbordone@obspm. fr \\ 2 GEPI, Observatoire de Paris, CNRS, Université Paris Diderot, Place Jules Janssen, 92190 Meudon, France \\ 3 INAF - Osservatorio Astronomico di Roma, via di Frascati 33, 00040 Monte Porzio Catone, Italy \\ 4 INAF - IASF, via del Fosso del Cavaliere 100, 00133 Rome, Italy \\ INAF - Osservatorio Astronomico di Trieste, via G. B. Tiepolo 11, 34131 Trieste, Italy
}

Received 12 December 2008 / Accepted 8 April 2009

\section{ABSTRACT}

\begin{abstract}
Context. The light elements $\mathrm{Li}, \mathrm{O}, \mathrm{Na}, \mathrm{Al}$, and $\mathrm{Mg}$ are known to show star-to-star variations in the globular clusters 47 Tuc and NGC 6752. Such variations are interpreted as coming from processing in a previous generation of stars.

Aims. In this paper we investigate the abundances of the $\alpha$-element sulfur, for which no previous measurements exist. In fact this element has not been investigated in any Galactic globular cluster so far. The only globular cluster for which such measurements are available is Terzan 7, which belongs to the Sgr dSph.

Methods. We use high-resolution spectra of the S I Mult. 1, acquired with the UVES spectrograph at the $8.2 \mathrm{~m}$ VLT-Kueyen telescope, for turn-off and giant stars in the two globular clusters. The spectra were analysed making use of ATLAS static plane parallel model atmospheres and SYNTHE spectrum synthesis. We also compute 3D corrections from $\mathrm{CO}^{5} \mathrm{BOLD}$ hydrodynamic models and apply corrections due to NLTE effects taken from the literature.

Results. In the cluster NGC 6752 sulfur has been measured only in four subgiant stars. We find no significant star-to-star scatter and a mean $\langle[\mathrm{S} / \mathrm{Fe}]\rangle=+0.49 \pm 0.15$, consistent with what is observed in field stars of the same metallicity. In the cluster 47 Tuc we measured $\mathrm{S}$ in 4 turn-off and 5 subgiant stars with a mean $\langle[\mathrm{S} / \mathrm{Fe}]\rangle=+0.18 \pm 0.14$. While this result is compatible with no star-to-star scatter we notice a statistically significant correlation of the sulfur abundance with the sodium abundance and a tentative correlation with the silicon abundance.

Conclusions. The sulfur-sodium correlation is not easily explained in terms of nucleosynthesis. An origin due to atomic diffusion can be easily dismissed. The correlation cannot be easily dismissed either, in view of its statistical significance, until better data for more stars is available.
\end{abstract}

Key words. stars: abundances - Galaxy: globular clusters: individual: NGC 104 - Galaxy: globular clusters: individual: NGC 6752

\section{Introduction}

There is substantial consensus that the so-called $\alpha$-elements $(\mathrm{O}$, $\mathrm{Ne}, \mathrm{Mg}, \mathrm{Si}, \mathrm{S}, \mathrm{Ar}, \mathrm{Ca}$, and $\mathrm{Ti}$ ) are synthesized essentially during hydrostatic and explosive burning phases in massive stars, and then released in the interstellar medium when such stars undergo type II supernova (SN) explosion (Chieffi \& Limongi 2004). As such, $\alpha$-elements provide crucial information for reconstructing the star formation history of stellar populations. Oxygen, $\mathrm{Mg}$, $\mathrm{Si}, \mathrm{Ca}$, and $\mathrm{Ti}$ are easily measured in stellar spectra and are thus the most frequent choice in stellar abundance studies. Sulfur, on the other hand, is the natural choice when abundances are determined in the interstellar medium (ISM), planetary nebulae (PN), or extragalactic absorption systems (e.g. damped Lyman alpha systems, DLA) due to the ease of measuring it and its weak tendency to form dust. The extent to which stellar and ISM/PN/DLA $\alpha$-element enrichments are comparable is thus dependent on whether or not $\mathrm{S}$ is supposed to vary in lockstep with the other $\alpha$-elements.

Sulfur abundances in Milky Way (MW) stars have been obtained by several authors in the last years (Nissen et al. 2007;

\footnotetext{
* Based on observations made with the ESO VLT-Kueyen telescope at the Paranal Observatory, Chile, in the course of the ESO-Large Programme 165.L-0263.
}

Caffau et al. 2007a; Ryde 2006; Caffau et al. 2005b; Korn \& Ryde 2005; Nissen et al. 2004; Ryde \& Lambert 2004, among the most recent), but agreement on its behavior at low metallicities has not been reached yet. While some of the cited authors (e.g. Nissen et al. 2007) find that $\mathrm{S}$ follows the same trend as other $\alpha$-elements ( $[\mathrm{S} / \mathrm{Fe}]$ showing a plateau at low metallicities), others point towards a steady growth in $[\mathrm{S} / \mathrm{Fe}]$ with decreasing metallicity (Israelian \& Rebolo 2001; Takada-Hidai et al. 2002), or an increase in the scatter of $[\mathrm{S} / \mathrm{Fe}]$ at low $[\mathrm{Fe} / \mathrm{H}]$ (Caffau et al. $2005 \mathrm{~b}$ ). The cited debate can in fact be extended to the overall behavior of $\alpha$-elements. While the standard view of $[\alpha / \mathrm{Fe}]$ evolution with metallicity has for some time now assumed the presence of a plateau below $[\mathrm{Fe} / \mathrm{H}] \sim-1$ (McWilliam 1997), both observations and models suggest the possibility of a continuing rise down to the lowest observed metallicities, at least for Mg and O (first pointed out by Abia \& Rebolo 1989; and more recently by François et al. 2004; and González Hernández et al. 2008).

It is perhaps surprising that sulfur has been studied up to now almost exclusively in field stars. To our knowledge, the only analysis of the $\mathrm{S}$ abundance in globular clusters is also the only one for extragalactic stars (Caffau et al. 2005a, in Terzan 7, one of the globular clusters of the Sagittarius dwarf Spheroidal Galaxy), where it behaves consistently with the rest of the 
$\alpha$-elements studied in the cluster, showing a low ratio with iron typical of dwarf spheroidal systems (Tautvaišienè et al. 2004; Sbordone et al. 2007). But on the other hand, S measurements are totally missing in MW globular clusters (GC). Globular clusters are somewhat peculiar environments regarding chemical enrichment, displaying some peculiar abundance anomalies and abundance correlations that have never been found elsewhere (for a review see Gratton et al. 2004). Among them the most well known are likely the so-called $\mathrm{O}-\mathrm{Na}$ anticorrelation, which appears to be widespread in MW globular cluster stars, and the $\mathrm{Mg}-\mathrm{Al}$ anticorrelation. The nucleosynthetic processes responsible for this are believed to be understood, since the chemical yields match the ones derived from p-capture processes occurring in (or near) regions where $\mathrm{H}$ is being burned via the $\mathrm{CNO}$ cycle at sufficiently high temperatures (Gratton et al. 2004). On the other hand, such a condition is reached during different phases of stellar evolution, so that the actual processing site is still debated, as is the mechanism through which the enriched gas is dispersed inside the cluster (Ventura et al. 2002; D'Antona et al. 2005; Decressin et al. 2007). There is nevertheless some consensus that the yields should be released through slow-moving ejecta, thus allowing the GC gravitational potential to retain them more easily.

We present here the first sulfur abundances in the GC 47 Tuc and NGC 6752, both of which display evident $\mathrm{Na}-\mathrm{O}$ and $\mathrm{Mg}-\mathrm{Al}$ abundance anticorrelations. They are also the only two clusters where an Na-Li anticorrelation has also been observed (Pasquini et al. 2005; Bonifacio et al. 2007).

\section{Observational data}

The spectra analyzed in this paper have been acquired in the course of the ESO-Large Programme 165.L-0263. Observations, atmospheric parameters, and abundances for elements other than S have been presented in Gratton et al. (2001), Carretta et al. (2004), and Bonifacio et al. (2007). We are only concerned here with the dichroic \#2, CD4 cross-disperser UVES@ VLT (Dekker et al. 2000) spectra (setting centered at $760 \mathrm{~nm}$ ). For most observations, the slit width was of $1^{\prime \prime}$ for a resolution of $R \sim 43000$. For the purpose of the sulfur measurement, only upper-red CCD data were used. Typical signal-to-noise ratios $(S / N)$ in the range were of about 15-20 per resolution element. Sulfur abundances were determined for nine stars observed in 47 Tuc, four of them turn off (TO) stars, 5 subgiants (SG). Four stars, all subgiants, were considered in NGC 6752. Typical exposure times were $1 \mathrm{~h}$ per star for NGC 6752 SG stars, $2 \mathrm{~h}$ per stars for 47 Tuc SG and $4 \mathrm{~h}$ per stars (in both cases performing multiple $1 \mathrm{~h}$ exposures) for 47 Tuc TO stars. An example of S I mult. 1 region is shown in Fig. 1.

\section{Analysis}

The atmospheric parameters have been taken from Gratton et al. (2001) for the stars in NGC 6752 and from Carretta et al. (2004) and Bonifacio et al. (2007) (for star \# 952) for the stars in 47 Tuc. The 47 Tuc stars have a typical temperature of $5800 \mathrm{~K}$ for the TO stars $(\log g=4.05)$ and of $5100 \mathrm{~K}$ for the subgiants $(\log g=3.84)$. The four NGC 6752 share the same atmosphere parameters, $T_{\text {eff }}=5347 \mathrm{~K}, \log g=3.54$. For each star we computed a model atmosphere using version 9 of the ATLAS code (Kurucz 1993, 2005) running under Linux (Sbordone 2005; Sbordone et al. 2004). We used the updated opacity distribution functions of Castelli \& Kurucz (2003) with microturbulent

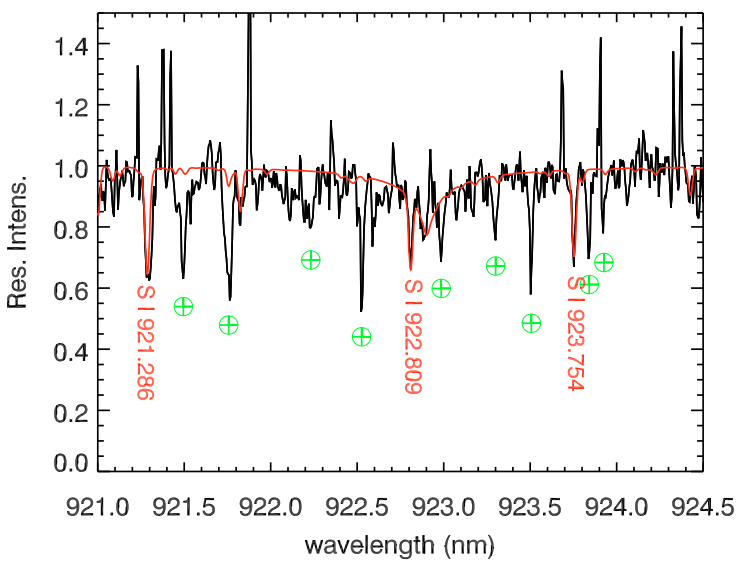

Fig. 1. The (normalized) spectrum of 47 Tuc star \# 1012 with superimposed a synthetic spectrum with $T_{\text {eff }} 5832 \mathrm{~K}, \log g 4.05,[\mathrm{Fe} / \mathrm{H}]=-0.5$. The three lines of S I mult. 1 are labeled, as well as the most prominent telluric absorptions (marked with $\oplus$ ).

Table 1. Sulfur line-by-line abundances for the program stars with wavelengths and $\log g f$.

\begin{tabular}{rcrr}
\hline \hline$\lambda$ & $921.286 \mathrm{~nm}$ & $922.809 \mathrm{~nm}$ & $923.754 \mathrm{~nm}$ \\
$\log g f$ & 0.42 & 0.26 & 0.04 \\
\hline NGC 6752 & & & \\
1406 & 5.80 & 6.07 & 6.04 \\
1665 & - & 6.35 & 6.29 \\
1461 & - & 6.35 & 6.37 \\
1481 & - & 6.35 & 6.36 \\
200613 & - & 6.23 & 5.63 \\
4661 & - & 5.62 & 5.87 \\
4907 & - & 5.63 & 5.85 \\
5048 & - & 5.81 & - \\
\hline 47 Tuc & & & \\
1012 & - & 6.56 & - \\
1081 & - & 6.68 & - \\
952 & - & 6.63 & - \\
975 & - & 6.72 & - \\
201075 & 6.65 & 6.86 & 6.43 \\
206415 & 6.73 & - & - \\
429 & - & 6.87 & - \\
433 & - & 6.87 & - \\
482 & - & 6.80 & - \\
\hline
\end{tabular}

velocity of $1 \mathrm{~km} \mathrm{~s}^{-1}$ and enhancement of $\alpha$-elements. The synthetic spectra were computed using the SYNTHE suite (Kurucz 1993, 2005) running under Linux (Sbordone 2005; Sbordone et al. 2004).

The strongest lines of S I in the optical range are those of Mult. 1 (three lines around $923 \mathrm{~nm}$ ) and of Mult. 6 (two lines at $869 \mathrm{~nm}$, see Table 1 ). We were unable to convincingly detect the lines of Mult. 6 in any of our spectra, so we did not even search for Mult. 8 (around $675 \mathrm{~nm}$ ), which is weaker. Our analysis is therefore based exclusively on the lines of Mult. 1. We adopt the $\log g f$ values of the NIST database, which holds the values of Wiese et al. (1969), which are experimental but D quality, corresponding to a possible error up to $50 \%$ (see Table 1 for the values). A thorough discussion of the available values for S I atomic data is avilable Caffau et al. (2005b).

The lines are in a spectral range contaminated by telluric absorption, so that they are rarely all free of telluric contamination. Moreover, due to the low metallicity of the two clusters examined and to the low $S / N$ of the spectra, in most cases, only one of the $\mathrm{S}$ I lines of Mult. 1 is detectable. Due to the radial velocity of 
the clusters, the line that happens to be the most often unblended is the one at $922.8 \mathrm{~nm}$, that is, the line laying on the wing of the hydrogen Paschen $\zeta$ line. The measurement of the equivalent width (EW) of lines falling on the wings of broad features is not easy, and produces a value that is usually lower than the true EW by about $5 \%$ (Caffau et al. 2005b). To determine the sulfur abundance a line profile fitting procedure is to be preferred taking the profile of the Paschen $\zeta$ into account. The two other lines of Mult. 1 are far enough from the Paschen $\zeta$ not to be affected by it, so that the EW measurement would be a good choice for these lines. To keep the analysis as homogeneous as possible, we nevertheless decided to determine the sulfur abundance using line profile fitting for all the unblended features. Such fitting was performed by means of the code described in Caffau et al. (2005b). In the fitting procedure, the line broadening was kept fixed at the instrumental level $\left(7 \mathrm{~km} \mathrm{~s}^{-1}\right)$. At this resolution, and with this $S / N$, typical macroturbulent and/or rotational broadening would not affect the measurement significantly. We discarded the lines contaminated by telluric absorption because the telluric line subtraction from a spectrum decreases the $S / N$, which is already low. Also, we did not possess fast rotator spectra taken at the same time as the science exposures, which would have required rescaling the telluric features, an operation that would add further uncertainty to the measurement. Line-by-line $\mathrm{S}$ abundances are listed in Table 1.

The S I lines of Mult. 1 are known to suffer from departures from LTE (Takeda et al. 2005). We therefore applied the NLTE corrections, interpolated in Table 2 of Takeda et al. (2005) to take this effect into account. For our programme stars these are all negative and range between -0.1 and -0.3 dex.

\subsection{Estimation of analysis uncertainties}

Uncertainty in the $\mathrm{S}$ abundance determination stems from both the limited $S / N$ of the spectra and the systematics of the analysis. To determine the effect of $S / N$, we followed the same method as described in Caffau et al. (2005b), namely by performing Monte Carlo simulations. In that paper the error estimates are obtained for a sample of dwarf stars with $[\mathrm{Fe} / \mathrm{H}]=-1.5$. We performed additional Monte Carlo tests to assess the $T_{\text {eff }}$ sensitivity of the $\mathrm{S}$ lines in the SG stars ( $T_{\text {eff }}$ being the only parameter showing a significant difference between TO and SG stars) and to ascertain that such uncertainties apply to the more metal-rich objects of 47 Tuc and came up with values that are essentially identical to the ones in Caffau et al. (2005b), which we assume from now on.

Concerning random errors, the result of a 1000-events simulation, for a parameter set compatible with the TO star set, indicates an $1 \sigma$ uncertainty (for a single line) of 0.12 dex for $S / N=30$, and $0.22 \operatorname{dex}$ for $S / N=15$. The same values hold for the SG stars. Given the typical $S / N$ of our spectra, we henceforth assume a typical uncertainty of 0.2 dex due to the $S / N$.

For the TO sample, a change in $\log g$ of \pm 0.25 dex causes a \pm 0.08 dex variation in $A(\mathrm{~S}), \pm 100 \mathrm{~K}$ in temperature causes a variation of $\mp 0.06 \mathrm{dex}$ in $A(\mathrm{~S})$, while $[\mathrm{Fe} / \mathrm{H}] \pm 0.2 \mathrm{dex}$ induces a \pm 0.03 dex variation in $A(S)$. Comparing with Table 3 of Carretta et al. (2004) one notices that both $T_{\text {eff }}$ and $\log g$ variations act in opposite senses on $[\mathrm{Fe} / \mathrm{H}]$ and on $A(\mathrm{~S})$. On the one hand, $\log g$ has a maximum stated error in Carretta et al. (2004) of 0.08 dex. Taking both the effect on metallicity and the one on $A(\mathrm{~S})$ into account, this would lead to a variation of $0.03 \mathrm{dex}$ in $[\mathrm{S} / \mathrm{Fe}]$, negligible in the scope of the present paper.

On the other hand, Carretta et al. (2004) claim a maximum $T_{\text {eff }}$ error of $\pm 90 \mathrm{~K}$ for TO stars and $\pm 60 \mathrm{~K}$ for subgiants. Shifting the whole temperature scale rigidly would not make or break the
[S/Fe] spread we observe, but one could argue that the Carretta et al. (2004) $T_{\text {eff }}$ scale might be excessively "compressed". By lowering the TO stars temperature and raising the one of the subgiants by the maximum amount stated, one would significantly reduce the observed $[\mathrm{S} / \mathrm{Fe}]$ spread. A $90 \mathrm{~K}$ lower temperature for the TO stars would lower their $[\mathrm{Fe} / \mathrm{H}]$ by 0.08 dex. The effect on $\mathrm{S}$ would then stem both from the reduced $T_{\text {eff }}$ and from the decreased metallicity, the total effect being roughly $\Delta A(\mathrm{~S})=+0.04$ dex. A similar calculation for the $\mathrm{SG}$ stars leads to $\Delta A(\mathrm{~S})=-0.03$, which becomes $\Delta[\mathrm{S} / \mathrm{Fe}]=+0.12$ for the $\mathrm{TO}$ and -0.09 for the $\mathrm{SG}$. This would reduce the $[\mathrm{S} / \mathrm{Fe}]$ spread by 0.21 dex, eliminating something less than half of it. On the other hand, such a change would lead $[\mathrm{Fe} / \mathrm{H}]$ to differ between $\mathrm{TO}$ and SG stars. Currently, the two subgroups give precisely the same metallicity $(-0.67)$ with a dispersion of 0.02 dex among TO stars and 0.01 dex among SG stars. Applying the aforementioned opposite temperature differences would separate the TO and SG stars by 0.14 dex in metallicity, roughly five times the quadratic sum of the two group uncertainties. We therefore suggest that any $T_{\text {eff }}$ bias is very likely to be a simple additive shift to both TO and SG star temperature. It is also worth noting that some imbalance ( 0.13 dex) exists between Fe I and Fe II abundances in the giants sample of Carretta et al. (2004). According to Carretta and collaborators' error budget, this would be entirely solved by rigidly increasing the $T_{\text {eff }}$ in subgiants by $100 \mathrm{~K}$. Again, a rigid shift of the $T_{\text {eff }}$ scale would affect neither the spread in $A(\mathrm{~S})$ nor in $[\mathrm{S} / \mathrm{Fe}]$, and would also break the ionization equilibrium in the TO sample. Shifting the giant sample alone would reduce the spread in sulfur abundance, but generate an Fe abundance spread. Besides, any increase in the subgiants $T_{\text {eff }}$ would mainly affect Fe I, raising its abundance. A discrepancy already exists between Carretta et al. (2004) and Koch \& McWilliam (2008) [Fe/H] scales, Koch \& McWilliam (2008) deriving an average $[\mathrm{Fe} / \mathrm{H}]$ lower by 0.09 dex. Such a discrepancy would be worsened, should the temperature for the Carretta et al. (2004) subgiants be increased.

\section{2. $3 D$ corrections}

We computed 3D corrections for the S I $922.8 \mathrm{~nm}$ line, for a set of atmosphere models encompassing the parameters of our program stars. Assuming an equivalent width of $12 \mathrm{pm}$ (typical for the observed stars), we tabulate the difference between the abundance needed to reproduce the equivalent width $(\mathrm{EW})$ in the 3D CO ${ }^{5}$ BOLD model (Freytag et al. 2002; Wedemeyer et al. 2004) and the one needed when using the comparison $1 \mathrm{D}_{\mathrm{LHD}}$ model (Caffau et al. 2007b), computed using a microturbulence of $1.5 \mathrm{~km} \mathrm{~s}^{-1}$. As such, the 3D abundance is obtained by adding the correction to our 1D abundances. Table 2 reports the model parameters as well. The 3D corrections appear to be on average 0.15 dex and positive, and appear highly homogeneous across the explored parameter space. In particular, the differences in the corrections for TO and SG stars are small. They have not been added to our measurements in the table and in the figures, since comparison elements do not have 3D corrections applied in this and other studies.

\section{Results}

Our results are summarised in Tables 3 and 4. Star identifications, $\mathrm{Na}$, and $\mathrm{Mg}$ abundances in Table 3 come from Gratton et al. (2001). In Table 4, identifications, atmosphere parameters and abundances other than $\mathrm{S}$ come from Carretta et al. (2004), with the exception of star \# 952, described in Bonifacio et al. (2007). other abundances coming from these 
Table 2. 3D corrections for the S I $922.8 \mathrm{~nm}$ line.

\begin{tabular}{lrrc}
\hline \hline$T_{\text {eff }}$ & $\log g$ & {$[\mathrm{Fe} / \mathrm{H}]$} & Corr. \\
\hline 5000 & 4.0 & 0.0 & 0.141 \\
5000 & 4.0 & -1.0 & 0.140 \\
4920 & 3.5 & 0.0 & 0.146 \\
4930 & 3.5 & -1.0 & 0.167 \\
\hline
\end{tabular}

Table 3. Sulfur abundances for the subgiant stars in NGC 6752.

\begin{tabular}{rcrrr}
\hline \hline Star & $N^{a}$ & {$[\mathrm{~S} / \mathrm{Fe}]$} & {$[\mathrm{Na} / \mathrm{Fe}]$} & {$[\mathrm{Mg} / \mathrm{Fe}]$} \\
\hline 1406 & 3 & +0.19 & +0.02 & +0.20 \\
1665 & 2 & +0.54 & +0.10 & +0.10 \\
1461 & 2 & +0.58 & +0.29 & +0.10 \\
1481 & 2 & +0.57 & +0.54 & -0.07 \\
\hline
\end{tabular}

${ }^{a}$ Number of $\mathrm{S}$ lines used.

papers are always 1D-LTE values, with the exception of $\mathrm{Na}$, which had NLTE corrections applied according to Gratton et al. (1999). We adopted a solar sulfur abundance of $A(\mathrm{~S})=7.21$ in the $[\mathrm{S} / \mathrm{Fe}]$ determination.

In NGC 6752, the low metallicity $([\mathrm{Fe} / \mathrm{H}]=-1.43)$ meant we were only able to measure the sulfur abundance in the four subgiant stars. In the fainter TO stars, the $S / N$ was too low to detect the $\mathrm{S}$ I lines. From the four subgiants we derive $\langle[\mathrm{S} / \mathrm{Fe}]\rangle=$ $+0.49 \pm 0.15$. Our estimated error on the sulfur abundances is 0.2 dex, dominated by the noise in the observed spectra. Thus the dispersion around the mean is fully compatible with the errors. Moreover, $[\mathrm{S} / \mathrm{Fe}]$ in NGC 6752 is fully compatible with the value found among field stars at this metallicity (see Caffau et al. 2005b, Fig. 10).

For the more metal-rich cluster, 47 Tuc, we have been able to measure at least one S I line for all the stars. All three lines of Mult. 1 were measurable in star 201075 . The $[\mathrm{S} / \mathrm{Fe}]$ ratio varies by almost $0.5 \mathrm{dex}$, with an average value of $\langle[\mathrm{S} / \mathrm{Fe}]\rangle=0.18 \pm$ 0.14 . Given that the uncertainty in the $S$ abundance in each star is estimated at 0.2 dex, the observed spread can in fact simply reflect the measurement uncertainty. Moreover, as in the case of NGC 6752 , the mean $[\mathrm{S} / \mathrm{Fe}]$ is totally compatible with the value displayed by field stars of abundance comparable to 47 Tuc (Caffau et al. 2005b).

The cluster 47 Tuc is believed to display $\mathrm{Na}-\mathrm{O}$, as well as $\mathrm{Mg}$-Al abundance anti-correlations (Carretta et al. 2004). We thus decided to check for possible correlations between $\mathrm{S}$ and some of the cited elements. Sulfur has never been measured before in 47 Tuc but information can be obtained about other elements by comparing the measurements in Carretta et al. (2004) with the ones obtained by Brown \& Wallerstein (1992), Alves-Brito et al. (2005), and Koch \& McWilliam (2008). In Fig. $2[\mathrm{~S} / \mathrm{Fe}]$ is plotted against $[\mathrm{Na} / \mathrm{Fe}]$. Black filled circles represent our data, points surrounded by a slightly larger circle indicate TO stars, and every data point is flagged with the corresponding star number. The typical size for the error is also indicated. Brown \& Wallerstein (1992), Alves-Brito et al. (2005), and Koch \& McWilliam (2008) do not measure sulfur, but for reference we add their values for $[\mathrm{Na} / \mathrm{Fe}]$. The correlation between $\mathrm{Na}$ and $\mathrm{S}$ abundance is clearly apparent in our data, and a Kendall $\tau$ rank correlation test in fact determines that the likelihood that $[\mathrm{Na} / \mathrm{Fe}]$ and $[\mathrm{S} / \mathrm{Fe}]$ are correlated is $97.8 \%{ }^{1}$.

1 This is equivalent to saying that the likelihood of the null hypothesis is $2.2 \%$. This is the likelihood that random errors applied to otherwise uncorrelated distributions might produce a level of correlation $\left(\tau_{K}\right)$ equal or superior to the observed one.

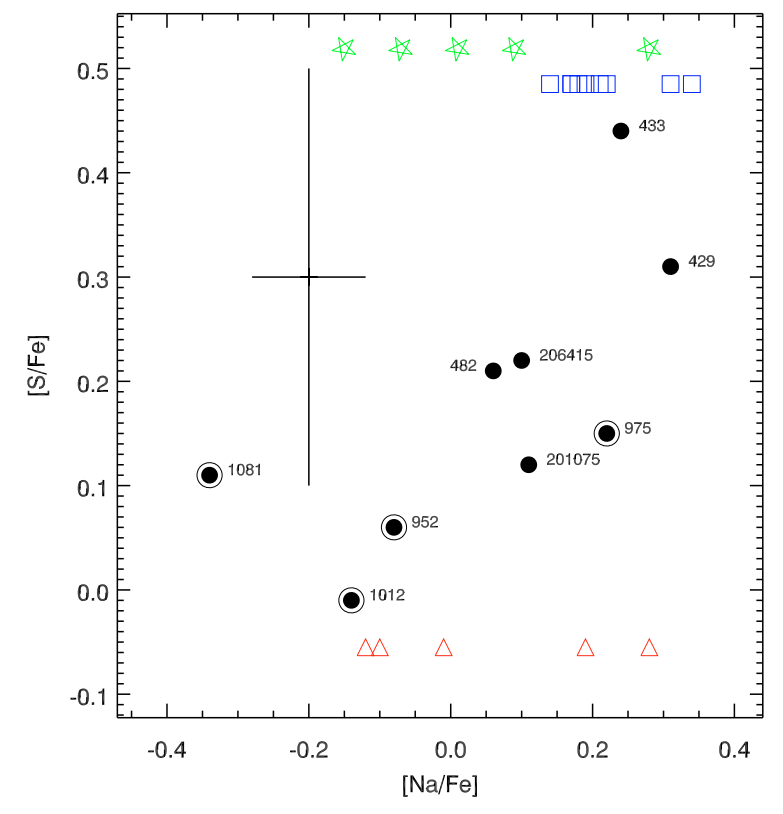

Fig. 2. $[\mathrm{S} / \mathrm{Fe}]$ plotted against $[\mathrm{Na} / \mathrm{Fe}]$ for our sample of 47 Tuc stars. $\mathrm{Na}$ spread is added for reference from Brown \& Wallerstein (1992) (open red triangles), Alves-Brito et al. (2005) (open green stars), and Koch \& McWilliam (2008) (open blue squares). A typical error for our dataset is also displayed. Stars in our sample belonging to the TO are identified by a larger open circle around them.

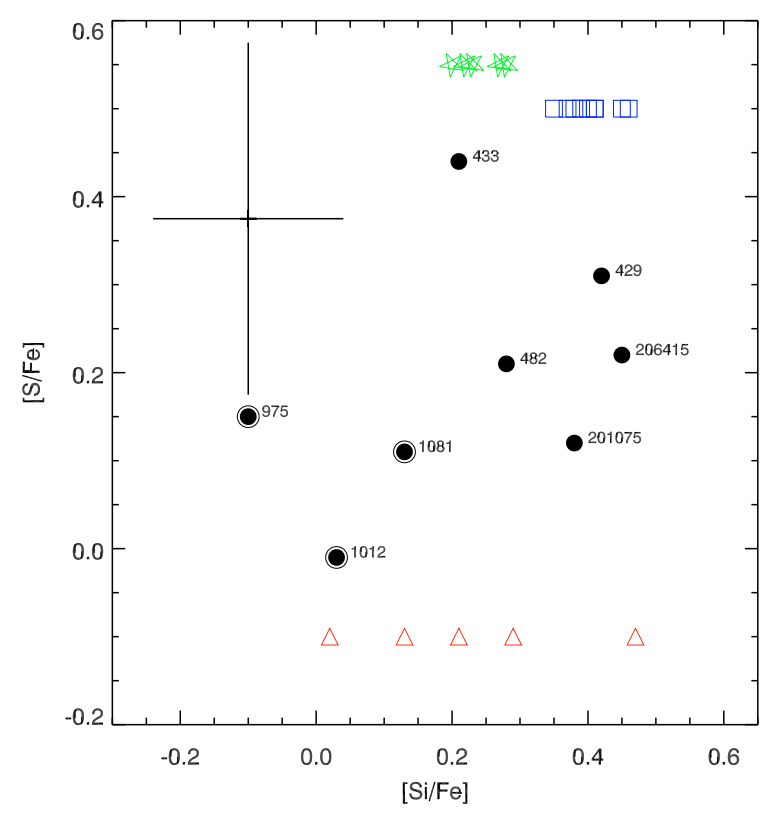

Fig. 3. $[\mathrm{S} / \mathrm{Fe}]$ plotted against $[\mathrm{Si} / \mathrm{Fe}]$ for our sample of 47 Tuc stars. $\mathrm{Si}$ spread is added for reference from Brown \& Wallerstein (1992) (open red triangles), Alves-Brito et al. (2005) (open green stars), and Koch \& McWilliam (2008) (open blue squares). A typical error for our dataset is also displayed. Stars in our sample belonging to the TO are identified by a larger open circle around them.

Another interesting thing to notice is how all the studies, with the exception of Koch \& McWilliam (2008), detect a significant spread in the $\mathrm{Na}$ abundance.

In Fig. 3 we instead plot $[\mathrm{S} / \mathrm{Fe}]$ versus [Si/Fe], all the symbols being the same as in Fig. 2. Again, there is an evident suggestion of a correlation, with the exception of star 433, which while having a very high $\mathrm{S}$ abundance, presents a less extreme 
Table 4. Sulfur abundances for the stars in 47 Tuc.

\begin{tabular}{rrrrrrrrr}
\hline \hline Star & $\begin{array}{r}T_{\text {eff }} \\
\mathrm{K}\end{array}$ & $\begin{array}{r}\log g \\
\text { cgs }\end{array}$ & $N^{a}$ & $\begin{array}{r}{[\mathrm{S} / \mathrm{Fe}]} \\
\text { dex }\end{array}$ & $\begin{array}{r}{[\mathrm{Fe} / \mathrm{H}]} \\
\text { dex }\end{array}$ & $\begin{array}{r}{[\mathrm{Na} / \mathrm{Fe}]} \\
\text { dex }\end{array}$ & $\begin{array}{r}{[\mathrm{Mg} / \mathrm{Fe}]} \\
\text { dex }\end{array}$ & $\begin{array}{r}{[\mathrm{Si} / \mathrm{Fe}]} \\
\text { dex }\end{array}$ \\
\hline 1012 & 5832 & 4.05 & 1 & -0.01 & -0.64 & -0.14 & +0.45 & +0.03 \\
1081 & 5832 & 4.05 & 1 & +0.11 & -0.64 & -0.34 & +0.50 & +0.13 \\
952 & 5832 & 4.05 & 1 & +0.06 & -0.64 & -0.08 & & \\
975 & 5832 & 4.05 & 1 & +0.15 & -0.64 & +0.22 & +0.21 & -0.10 \\
201075 & 5165 & 3.84 & 3 & +0.12 & -0.68 & +0.11 & +0.42 & +0.38 \\
206415 & 5112 & 3.84 & 1 & +0.22 & -0.70 & +0.10 & +0.34 & +0.45 \\
429 & 5081 & 3.84 & 1 & +0.31 & -0.65 & +0.31 & +0.39 & +0.42 \\
433 & 5106 & 3.84 & 1 & +0.44 & -0.78 & +0.24 & +0.49 & +0.21 \\
482 & 5090 & 3.84 & 1 & +0.21 & -0.62 & +0.06 & +0.64 & +0.28 \\
\hline
\end{tabular}

${ }^{a}$ Number of S lines used.

$[\mathrm{Si} / \mathrm{Fe}]$. Likelihood of the correlation from Kendall $\tau$ test is here about $79 \%$, but grows to $91 \%$ if star 433 is removed from the sample. By removing star 433 from the $[\mathrm{Na} / \mathrm{Fe}]$ vs. $[\mathrm{S} / \mathrm{Fe}]$ correlation test, one gets instead a slight decrease in the correlation likelihood, down to $94 \%$. It is worth noting how star 433 has, according to Carretta et al. (2004), an unusually low [Fe/H] ( 0.14 dex below the average, way beyond the scatter of the Fe I abundances) and an unsatisfactory Fe I-Fe II ionization equilibrium (0.21 dex difference). Since no evidence ever emerged that 47 Tuc shows a spread in iron abundance, one might regard these results as suggestive of some problem in the analysis of star 433. If we tentatively assign the average $\mathrm{Fe}$ abundance $([\mathrm{Fe} / \mathrm{H}]=-0.66)$ to star 433 , we obtain $[\mathrm{S} / \mathrm{Fe}]=0.32$, more in line with the rest of the sample. In fact, star 433 has an $[\mathrm{S} / \mathrm{H}]$ exceeding by just 0.14 dex the average value $(\langle[\mathrm{S} / \mathrm{H}]\rangle=-0.48$. $\left.[\mathrm{S} / \mathrm{H}]_{433}=-0.34\right)$.

Given that most low-S stars (and low-Na stars) are TO stars, it is worth investigating whether diffusion in the stellar atmosphere might be responsible for the observed abundance spread and its correlation with the $\mathrm{Na}$ abundance. One would in this case expect TO stars to be most affected by diffusion, while SG stars should no longer display them due to the deeper reach of their atmospheric convective zone. According to current models this is not a viable explanation of the observed spreads. According to Korn et al. (2007, Fig. 1), by using a model with radiative acceleration and turbulent mixing, an old $[\mathrm{Fe} / \mathrm{H}]=-2$ TO star shows photospheric depletion of about 0.25 dex in $\mathrm{Na}$, $0.20 \mathrm{dex}$ in $\mathrm{S}$ and $0.15 \mathrm{dex}$ in $\mathrm{Fe}$. The effect would be then $0.05 \mathrm{dex}$ on $[\mathrm{S} / \mathrm{Fe}]$ and $0.1 \mathrm{dex}$ in $[\mathrm{Na} / \mathrm{Fe}]$, too small to explain the observations.

\section{Discussion}

The sulfur abundance in NGC 6752 agrees with the general trend of $\alpha$-elements in the field, and does not present any significant spread. In 47 Tuc the situation is less clear. The presence of a significant correlation between the $\mathrm{S}$ and $\mathrm{Na}$ abundance, and the hint of an S/Si correlation, suggest that the $\mathrm{S}$ abundance spread might indeed be real.

Recently, Koch \& McWilliam (2008) in a thorough analysis of 47 Tuc based on high-quality spectra of bright giants find only a very limited spread in abundances, and are unable to confirm any significant spread in $\mathrm{O}, \mathrm{Na}, \mathrm{Al}$, and $\mathrm{Mg}$. Such discrepancy with the results of Brown \& Wallerstein (1992), Carretta et al. (2004), Alves-Brito et al. (2005), and Bonifacio et al. (2007) has to date no satisfactory explanation. This is not the venue to reassess the work of the cited authors in detail. Suffice it to say that, applying an error analysis similar to the one sketched above for

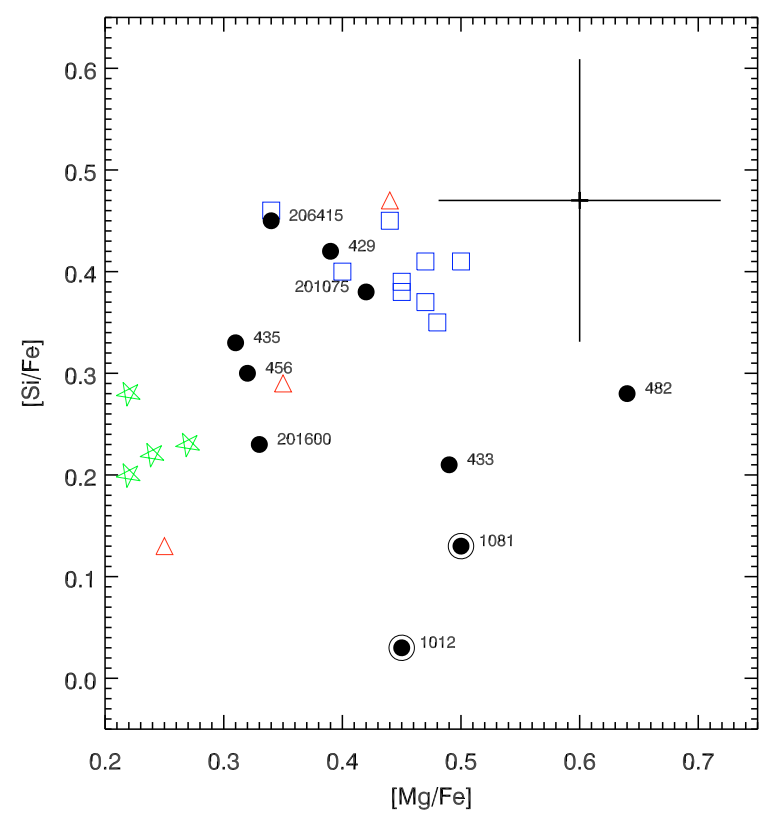

Fig. 4. $[\mathrm{Si} / \mathrm{Fe}]$ plotted against $[\mathrm{Mg} / \mathrm{Fe}]$ for our sample of 47 Tuc stars, plus Brown \& Wallerstein (1992) (open red triangles), Alves-Brito et al. (2005) (green stars), and Koch \& McWilliam (2008) (open blue squares). Stars in our sample belonging to the TO are identified by a larger open circle around them. A typical error for our sample is also displayed.

sulfur, the reality of the spreads in 47 Tuc, as well as their correlation appear difficult to dismiss.

In Fig. 4 we plot $[\mathrm{Si} / \mathrm{Fe}]$ versus $[\mathrm{Mg} / \mathrm{Fe}]$ for our stars (abundances are taken from Carretta et al. 2004), the sample of Brown \& Wallerstein (1992) and the one of Koch \& McWilliam (2008). Symbols are the same as in Figs. 2 and 3. The first thing that can be noted here is that, with the exception of star $482^{2}$, our sample and the one of Koch \& McWilliam (2008) in fact present the same spread in $[\mathrm{Mg} / \mathrm{Fe}]$, but a very different one in $[\mathrm{Si} / \mathrm{Fe}]$. A word of caution should be here given regarding silicon: it appears that $\mathrm{Si}$ shows a systematic discrepancy between TO and RGB in extremely metal poor stars (McWilliam 1997; Cohen et al. 2004; Bonifacio et al. 2009). The reasons for this discrepancy are not known, NLTE effects being a possibility. The sense and amount of the discrepancy is compatible with the one seen here, so the possibility exists that the Si spread is not real. On the other hand, such an effect has never been reported on

\footnotetext{
2 Star 482 is indeed suspect in a more general way, presenting the largest Fe I - Fe II imbalance of the whole Carretta et al. (2004) sample.
} 
stars as metal rich as the ones in 47 Tuc. Another element for which the various authors do not reach a satisfactory agreement is $\mathrm{Ca}$. Average $[\mathrm{Ca} / \mathrm{Fe}]$ is 0.34 in Koch \& McWilliam (2008), 0.18 in Carretta et al. (2004), but solar according to Brown \& Wallerstein (1992) and Alves-Brito et al. (2005).

Another important aspect is of course constituted by the existence of significant correlations among the abundances. One might consider as worrying that all the TO stars of the sample exhibit a low S abundance. On the other hand, star 201075, which is a subgiant, and the one star where all the three lines of Mult. 1 were measured, shows low S as well. By examining Table 1 one might notice that the line at $922.8 \mathrm{~nm}$ gives the highest abundance for star 201075. Since this line is the one most frequently measured in the other stars, one might object that, had it been the only one measured, the star would have assigned a higher abundance, compatible with the other subgiants. It does need to be noted that in NGC 6752 both this line and the one at $923.7 \mathrm{~nm}$ are systematically measured, and no sign exists of the latter giving a lower abundance. As explained in Sect. 3.1, the quoted $T_{\text {eff }}$ uncertainty should be sufficient to remove the spread between TO and subgiant stars, if TO star temperature was lowered and SGB star temperature increased. On the other hand, this would make star 201075 even more discrepant, and induce an Fe abundance spread between TO and SGB stars.

Furthermore, a Monte Carlo simulation of 1000 events has been performed to check the likelihood that a significant correlation might emerge in the $[\mathrm{S} / \mathrm{Fe}]$ vs. $[\mathrm{Na} / \mathrm{Fe}]$ plot when the quoted errors are superimposed to a constant $[\mathrm{S} / \mathrm{Fe}]$ for all the stars. Unsurprisingly, the result ( $2 \%$ likelihood) confirms the result of the Kendall $\tau$ null-hypothesis probability. It is very unlikely that random errors might have produced the observed $\mathrm{Na}-\mathrm{S}$ correlation.

In short, we believe that strong hints of an $\mathrm{S}$ abundance inhomogeneity in 47 Tuc do exist. It is nevertheless clear that the currently available data do not allow clarifying the matter, due to a combination of low statistics and significant uncertainties. Further study would thus be in order, specifically targeting a large number of stars from TO to RGB, and focusing on the elements that show significant spreads. Still, being unable to justify the current observations by means of either random or systematic uncertainties, we feel compelled to look for a nucleosynthetic explanation. The Na-O anticorrelation is usually explained by self pollution within the cluster by slow Na-rich winds, either from AGB stars or from massive rotating stars. In general, the stars of pristine cluster composition are expected to show low $\mathrm{Na}$ abundance, while a higher abundance is indication of "polluted" stars. Should Si and S abundance spreads be confirmed, and should they both correlate with the $\mathrm{Na}$ spread (which is quite uncertain in the Si case), this would pose at least two problems.

First, since Na-poor stars are both Si-poor and S-poor, the aforementioned model would designate them as the "unpolluted" stars. The 47 Tuc cluster would then have formed with an unusual low abundance of $\mathrm{Si}$ and $\mathrm{S}$, at variance e.g. with $\mathrm{Mg}$. Low $[\alpha / \mathrm{Fe}]$ ratios are indeed observed in dwarf galaxies, but typically attributed to environmental effects (e.g. Lanfranchi \& Matteucci 2007), and no evidence exists that they might privilege one explosive product (e.g. Si) over another (e.g. Mg).

Second, as just noted, both $\mathrm{Si}$ and $\mathrm{S}$ are believed to almost exclusively be products of SN II explosive burnings. Elementary models forbid globular clusters being chemically enriched by internal SN explosions, since the total released energy is comparable to the cluster binding energy, and even one single SN II explosion should remove all the available gas from the cluster potential well, and stop the star formation. This is why "slow wind" mechanisms have been invoked to produce e.g. the $\mathrm{Na}$ pollution. At the same time, the lack of any detectable spread in Fe abundance in 47 Tuc constitutes an additional problem, since one would expect some amount of Fe to be produced by the SN along with $\alpha$-elements. On the other hand, such simplistic considerations likely provide too crude a model of the actual phenomenon, since they assume a spherically symmetrical, central explosion whose energy is efficiently transferred to the cluster gas. SN II explosions are in fact rarely spherically symmetrical (Wang \& Wheeler 2008). Instead, they tend to release a large part of the energy along more or less collimated jets that are not efficient in transferring the energy to the cluster ISM. Moreover, $\mathrm{SN}$ that explode in a non-spherically symmetrical way tend to produce larger fallback, which would in turn limit their Fe yield, thus eliminating the "missing Fe spread" problem. It is also worth noting that detailed models of stellar superclusters foresee a destructive interference among shock waves from different supernovae, within a given critical radius (Wünsch et al. 2008). As a result, the inner part of the cluster would not only retain the yields, but also experience an accelerated star formation. Whether this might be the case on the smaller scale of normal GC (or whether some/all the GC might form as superclusters) is uncertain. Core collapse SN are a source of $\mathrm{Na}$ as well, and we see that the $\mathrm{Na}$ abundance correlates with the $\mathrm{S}$ abundance. The applicability of the aforementioned model thus relies on the (yet unexplored) possibility that high Na-yield and high $\alpha$-yield (or at least high S-yield) happen together.

An alternate way to produce $\mathrm{S}$ and $\mathrm{Si}$ would be through proton capture reactions. This is the same source invoked to explain the $\mathrm{Na}$ overabundance, which would be an obvious advantage. On the other hand, $\mathrm{S}$ should be produced this way through the ${ }^{31} \mathrm{P}(\mathrm{p}, \gamma){ }^{32} \mathrm{~S}$ reaction. The phosphorus abundance in 47 Tuc is unknown, and in general, knowledge about $\mathrm{P}$ production channels and abundances in stars are sketchy at best (see Caffau et al. 2007b, and references therein). However, P in the Sun is roughly 3 dex less abundant than S. If the same abundance ratio holds at lower metallicities, it would be impossible to produce any significant amount of $\mathrm{S}$ at expenses of P. Phosphorus should then be formed at expenses of $\mathrm{Si}$, which should then anticorrelate with $\mathrm{S}$, which is not the case. The next abundant enough seed is $\mathrm{Mg}(\mathrm{Mg} \rightarrow \mathrm{Al} \rightarrow \mathrm{Si} \rightarrow \mathrm{P} \rightarrow \mathrm{S})$, which indeed might show weak signs of anti-correlation with Si (Fig. 4), although they are below any statistical significance. Given the very scarce knowledge about $\mathrm{P}$ abundances and production mechanisms, one might even suppose that the "original" 47 Tuc $\mathrm{P}$ abundance is heavily enhanced, thus providing enough seeds to form $\sim 0.2 \mathrm{dex}$ of $\mathrm{S}$. The $\mathrm{P}$ abundance has never been measured in 47 Tuc, but its abundance has been determined very recently in horizontal branch (HB) stars in NGC 6397 and NGC 6752 (Hubrig et al. 2009), where it appears strongly enhanced, by more than 2 dex in NGC 6752, and almost 3 dex in NGC 6397. Chemical anomalies in HB stars are usually attributed to diffusion effects, but the current models (Michaud et al. 2008) do not seem to account for more than $\sim 0.7$ dex of $\mathrm{P}$ enhancement in the photospheres of HB stars of similar temperature. We thus think that the possibility of an enhancement of $\mathrm{P}$ abundance in globular clusters cannot be ruled out at the moment.

Acknowledgements. We whish to thank the anonymous referee for the useful comments that really helped to improve the paper. The authors L.S., H.-G.L., P.B. acknowledge financial support from EU contract MEXT-CT-2004-014265 (CIFIST). This research has made use of the SIMBAD database, operated at the CDS, Strasbourg, France, and of NASA's Astrophysics Data System. 


\section{References}

Abia, C., \& Rebolo, R. 1989, ApJ, 347, 186

Alves-Brito, A., Barbuy, B., Ortolani, S., et al. 2005, A\&A, 435, 657

Bonifacio, P., Pasquini, L., Molaro, P., et al. 2007, A\&A, 470, 153

Bonifacio, P., Pasquini, L., Molaro, P., et al. 2009, A\&A, submitted

Brown, J. A., \& Wallerstein, G. 1992, AJ, 104, 1818

Caffau, E., \& Ludwig, H.-G. 2007, A\&A, 467, L11

Caffau, E., Bonifacio, P., Faraggiana, R., \& Sbordone, L. 2005a, A\&A, 436, L9

Caffau, E., Bonifacio, P., Faraggiana, R., et al. 2005b, A\&A, 441, 533

Caffau, E., Faraggiana, R., Bonifacio, P., Ludwig, H.-G., \& Steffen, M. 2007a, A\&A, 470, 699

Caffau, E., Steffen, M., Sbordone, L., Ludwig, H.-G., \& Bonifacio, P. 2007b, A\&A, 473, L9

Carretta, E., Gratton, R. G., Bragaglia, A., Bonifacio, P., \& Pasquini, L. 2004, A\&A, 416, 925

Castelli, F., \& Kurucz, R. L. 2003, in Modelling of Stellar Atmospheres, ed. N. Piskunov, W. W. Weiss, \& D. F. Gray, IAU Symp., 210, 20P

Chieffi, A., \& Limongi, M. 2004, ApJ, 608, 405

Cohen, J. G., Christlieb, N., McWilliam, A., et al. 2004, ApJ, 612, 1107

D’Antona, F., Bellazzini, M., Caloi, V., et al. 2005, ApJ, 631, 868

Decressin, T., Charbonnel, C., \& Meynet, G. 2007, A\&A, 475, 859

Dekker, H., D’Odorico, S., Kaufer, A., Delabre, B., \& Kotzlowski, H. 2000, Proc. SPIE, 4008, 534

François, P., Matteucci, F., Cayrel, R., et al. 2004, A\&A, 421, 613

Freytag, B., Steffen, M., \& Dorch, B. 2002, Astron. Nachr., 323, 213

González Hernández, J. I., Bonifacio, P., Ludwig, H.-G., et al. 2008, A\&A, 480, 233

Gratton, R. G., Carretta, E., Eriksson, K., \& Gustafsson, B. 1999, A\&A, 350, 955

Gratton, R. G., Bonifacio, P., Bragaglia, A., et al. 2001, A\&A, 369, 87

Gratton, R., Sneden, C., \& Carretta, E. 2004, ARA\&A, 42, 385

Hubrig, S., Castelli, F., De Silva, G., et al. 2009 [arXiv: 0903 . 5182]

Israelian, G., \& Rebolo, R. 2001, ApJ, 557, L43
Koch, A., \& McWilliam, A. 2008, AJ, 135, 1551

Korn, A. J., \& Ryde, N. 2005, A\&A, 443, 1029

Korn, A. J., Grundahl, F., Richard, O., et al. 2007, ApJ, 671, 402

Kurucz, R. 1993, SYNTHE Spectrum Synthesis Programs and Line Data, Kurucz CD-ROM No. 18 (Cambridge, Mass.: Smithsonian Astrophysical Observatory)

Kurucz, R. L. 2005, Mem. Soc. Astron. It. Supp., 8, 14

Lanfranchi, G. A., \& Matteucci, F. 2007, A\&A, 468, 927

McWilliam, A. 1997, ARA\&A, 35, 503

Meynet, G., \& Maeder, A. 2002, A\&A, 390, 561

Michaud, G., Richer, J., \& Richard, O. 2008, ApJ, 675, 1223

Nissen, P. E., Chen, Y. Q., Asplund, M., \& Pettini, M. 2004, A\&A, 415, 993

Nissen, P. E., Akerman, C., Asplund, M., et al. 2007, A\&A, 469, 319

Pasquini, L., Bonifacio, P., Molaro, P., et al. 2005, A\&A, 441, 549

Ryde, N. 2006, A\&A, 455, L13

Ryde, N., \& Lambert, D. L. 2004, A\&A, 415, 559

Sbordone, L. 2005, Mem. Soc. Astron. It. Supp., 8, 61

Sbordone, L., Bonifacio, P., Castelli, F., \& Kurucz, R. L. 2004, Mem. Soc. Astron. It. Supp., 5, 93

Sbordone, L., Bonifacio, P., Marconi, G., Buonanno, R., \& Zaggia, S. 2005, A\&A, 437, 905

Sbordone, L., Bonifacio, P., Buonanno, R., et al. 2007, A\&A, 465

Takada-Hidai, M., Takeda, Y., Sato, S., et al. 2002, ApJ, 573, 614

Takeda, Y., Hashimoto, O., Taguchi, H., et al. 2005, PASJ, 57, 751

Tautvaišienė, G., Wallerstein, G., Geisler, D., Gonzalez, G., \& Charbonnel, C. 2004, AJ, 127, 373

Ventura, P., D’Antona, F., \& Mazzitelli, I. 2002, A\&A, 393, 215

Wang, L., \& Wheeler, J. C. 2008, ARA\&A, 46, 433

Wedemeyer, S., Freytag, B., Steffen, M., Ludwig, H.-G., \& Holweger, H. 2004, A\&A, 414, 1121

Wiese, W. L., Smith, M. W., \& Miles, B. M. 1969, NSRDS-NBS, Washington, D.C.: US Department of Commerce, National Bureau of Standards

Woosley, S. E., \& Heger, A. 2007, Phys. Rep., 442, 269

Woosley, S., \& Janka, T. 2005, Nature Physics, 1, 147

Wünsch, R., Tenorio-Tagle, G., Palouš, J., \& Silich, S. 2008, ApJ, 683, 683 